\title{
Características clínicas, gravedad y control del asma en Colombia: un análisis post hoc del estudio ASLA (Asthma control in Latin America)
}

Juliana Beltrán ${ }^{1}$, Andrés González ${ }^{1}$, Felipe Moraes ${ }^{2}$, Rafael Alfonso-Cristancho ${ }^{3}$, Valentina Di Boscio ${ }^{4}$, Claudia Soares ${ }^{2}$ (1) GSK Colombia, (2) GSK Brasil, (3) GSK Global, (4) GSK Argentina

Introducción

El asma es la segunda enfermedad respiratoria crónica con mayor impacto en el sistema de salud en Colombia (1). La frecuencia de asma grave y no controlada es aún incierta.

Objetivo

Describir las caracteristicas clínicas, uso de recursos y costos directos de la atención de EPOC en una institución prestadora de salud de Bogota.

Métodos

- Diseño: ASLA (2) fue un estudio transversal multicéntrico conducido en Argentina, Chile, Colombia y México entre 2013 y 2015.

Población: Se incluyeron pacientes $\geq 12$ años con diagnóstico de asma atendidos en centros ambulatorios especializados, seguidos por neumólogo y con al menos una visita y prescripción para el tratamiento de la enfermedad en los últimos seis meses. Los datos se obtuvieron mediante entrevista clínica. Este análisis post hoc describe a la subpoblación de pacientes $\geq 18$ años reclutados en los tres centros participantes en Colombia.

- Información clínica: Se usó una definición operativa de asma grave como la necesidad de tratamiento pasos 405 de GINA (uso de corticosteroide inhalado a dosis altas más un segundo controlador, corticosteroides orales u omalizumab) a partir de criterios ERS/ATS 2014 modificados (3). Se definió asma no controlada como un puntaje $<20$ en el Asthma Control Test (ACT) y exacerbación grave como una visita a urgencias hospitalización causadas por empeoramiento de los sintomas.

\section{Resultados}

$\checkmark$ El estudio ASLA incluyó 113 pacientes en Colombia, 53,1\% con terapia pasos 405

$\checkmark$ Del total, un $67,3 \%$ de los pacientes tenían asma no controlada y $31,8 \%$ reportaron dos o más exacerbaciones graves en el último año.

$\checkmark$ Los dos grupos según tratamiento fueron cualitativamente similares, excepto por edad, sexo, visitas a urgencias, hospitalizaciones y exacerbaciones por asma.

\section{Conclusiones}

Una proporción importante de los pacientes tratados en las instituciones especializadas del estudio ASLA en Colombia tienen tratamiento para asma grave y enfermedad no controlada según los criterios ERS/ATS ACT respectivamente.

Punto de discusión

Bajo tamaño de muestra, errores de clasificación o el efecto del tratamiento recibido pueden explicar las similitudes clínicas halladas entre los grupos.

\begin{tabular}{|c|c|c|c|c|c|c|c|c|c|c|}
\hline \multicolumn{4}{|c|}{ Tabla 1: Características demográficas y clínicas } & \multicolumn{7}{|c|}{ Tabla 2: Frecuencia de comorbilidades reportadas } \\
\hline Variable & $\begin{array}{r}\text { Pasos 1-3 } \\
\text { [Asma no grave] } \\
(n=53)\end{array}$ & $\begin{array}{r}\text { Pasos 4-5 } \\
\text { [Asma grave] } \\
(n=60)\end{array}$ & $\begin{array}{r}\text { Total } \\
(n=113)\end{array}$ & & [Asm & $\begin{array}{l}\text { Pasos 1-3 } \\
\text { no grave] } \\
(n=53)\end{array}$ & & $\begin{array}{r}\text { Pasos 4-5 } \\
\text { [Asma grave] } \\
(n=60)\end{array}$ & & $\begin{array}{r}\text { Total } \\
(n=113)\end{array}$ \\
\hline \multirow{2}{*}{ Edad } & \multirow{2}{*}{$46,5(15,7)$} & \multirow{2}{*}{$51,2(14,5)$} & $\begin{array}{r}49,0 \\
(15,2)\end{array}$ & \multirow{2}{*}{$\begin{array}{l}\text { Alteraciones hormonales } \\
\text { Apnea obstructiva del sueño }\end{array}$} & \multicolumn{2}{|c|}{$7(13,2)$} & \multirow{2}{*}{\multicolumn{2}{|c|}{$\begin{array}{l}8(13,3) \\
7(11,7)\end{array}$}} & \multirow{2}{*}{\multicolumn{2}{|c|}{$\begin{array}{l}15(13,3) \\
15(13,3)\end{array}$}} \\
\hline & & & 28,8 & & \multicolumn{2}{|r|}{$8(15,1)$} & & & & \\
\hline Edad al dlagnóstlco & $27,7(18,8)$ & $29,7(20,8)$ & $(19,9)$ & Arritmia cardiaca & \multirow{2}{*}{\multicolumn{2}{|c|}{$\begin{array}{l}3(5,7) \\
2(3,8)\end{array}$}} & \multirow{2}{*}{\multicolumn{2}{|c|}{$\begin{array}{l}2(3,3) \\
3(5,0)\end{array}$}} & & $5(4,4)$ \\
\hline Sexo femenino & $39(73,6)$ & $48(80,0)$ & $87(77,0)$ & Cáncer & & & & & & $5(4,4)$ \\
\hline Estado nutricional * & & & & \multirow[b]{2}{*}{ Enfermedad articular degenerativa } & \multicolumn{2}{|r|}{$\begin{array}{r}2(3,8) \\
6(11,3)\end{array}$} & \multicolumn{2}{|r|}{$\begin{array}{l}3(5,0) \\
4(6,7)\end{array}$} & & $10(8,9)$ \\
\hline Bajo peso & $2(3,80)$ & $1(1,7)$ & $3(2,7)$ & & \multirow{2}{*}{\multicolumn{2}{|c|}{$2(3,8)$}} & \multicolumn{2}{|r|}{$8(13,3)$} & & $10(8,9)$ \\
\hline Eutrófico & $21(39,6)$ & $24(40,0)$ & $45(39,8)$ & Enfermedad cerebrovascular & & & \multicolumn{2}{|r|}{$0(0,0)$} & & $1(0,9)$ \\
\hline Sobrepeso & $22(41,5)$ & $24(40,0)$ & $46(40,7)$ & Alteraciones psicológicas & \multirow{2}{*}{\multicolumn{2}{|c|}{$13(24,5)$}} & \multicolumn{2}{|r|}{$15(25,0)$} & & $28(24,8)$ \\
\hline Obeso & $8(15,1)$ & $10(16,7)$ & $18(15,9)$ & Enfermedad pulmonar obstructiva crónica & \multirow{2}{*}{\multicolumn{2}{|c|}{$9(17,0)$}} & & $11(18,3)$ & & $20(17,7)$ \\
\hline Tabaqulsmo actual o prevlo & $8(15,1)$ & $5(8,3)$ & $13(11,5)$ & $\begin{array}{l}\text { Entermedad purmonar osstructiva cronica } \\
\text { Falla cardiaca congestiva }\end{array}$ & & & & $1(1,7)$ & & $2(1,8)$ \\
\hline Puntaje ACT total & $16,8(4,9)$ & $16,1(5,4)$ & $16,4(5,2)$ & $\begin{array}{l}\text { Falla cardiaca congestiva } \\
\text { Hipertensión }\end{array}$ & & $11(20,8)$ & & $12(20,0)$ & & $23(20,4)$ \\
\hline Asma no controlada $(A C T<20)$ & $37(69,8)$ & $39(65,0)$ & $76(67,3)$ & Infecciones respiratorias recurrentes & & $12(22,7)$ & & $22(36,7)$ & & $34(30,1)$ \\
\hline $\begin{array}{l}\text { Una o más visittas a urgencias } \\
\text { por asma }\end{array}$ & $25(47,2)$ & $29(48,3)$ & $54(47,8)$ & $\begin{array}{l}\text { Intecciones respiratorras recurrentes } \\
\text { Enfermedad cardiaca isquémica }\end{array}$ & & $\begin{array}{r}12(22,7) \\
2(3,8)\end{array}$ & & $\begin{aligned} 22(36,7) \\
3(5,0)\end{aligned}$ & & $\begin{array}{r}34(30,1) \\
5(4,4)\end{array}$ \\
\hline Visitas a urgencias por asma & & & & Obesidad & & $9(17,0)$ & & $14(23,3)$ & & $23(20,4)$ \\
\hline & $28(52,8)$ & $31(51,7)$ & $59(52,2)$ & Reflujo gastroesofágico & & $25(47,2)$ & & $27(45,0)$ & & $52(46,0)$ \\
\hline 1 & $10(18,9)$ & $12(20,0)$ & $22(19,5)$ & Rinitis crónica & & $36(67,9)$ & & $39(65,0)$ & & $75(66,4)$ \\
\hline 2 & $6(11,3)$ & $3(5,0)$ & $9(8,0)$ & Sinusitis crónica & & $17(32,1)$ & & $21(35,0)$ & & $38(33,6)$ \\
\hline 3 & $5(9,4)$ & $3(5,0)$ & $8(7,1)$ & Datos expresados como frecuencia (porcentaje) & & & & & & \\
\hline 4 o más & $4(7,6)$ & $11(18,3)$ & $15(13,3)$ & & & & & & & \\
\hline $\begin{array}{l}\text { Una o más hospitallzaciones por } \\
\text { asma }\end{array}$ & $8(15,1)$ & $6(10,0)$ & $14(12,4)$ & & & & & & & \\
\hline Hospitalizaciones por asma & & & & Tabla 3: Frecue & cia de eve & tos clínic & de inter & & & \\
\hline $\begin{array}{l}0 \\
1 \\
2 \\
3\end{array}$ & $\begin{array}{r}45(84,9) \\
1(1,9) \\
4(7,6) \\
0(0,0)\end{array}$ & $\begin{array}{r}54(90,0) \\
1(1,7) \\
3(5,0) \\
1(1,7)\end{array}$ & $\begin{array}{r}99(87,6) \\
2(1,8) \\
7(6,2) \\
1(0,9)\end{array}$ & & $\begin{array}{r}\text { Pasos } \\
\text { [Asma n } \\
(\mathrm{n}=\end{array}$ & $\begin{array}{l}1-3 \\
\text { grave] } \\
\text { 3) }\end{array}$ & $\begin{array}{r}\text { Pasos } \\
\text { [Asma } \\
\text { (n= }\end{array}$ & $\begin{array}{l}4-5 \\
\text { rrave] } \\
\text { s0) }\end{array}$ & $\begin{array}{r}\text { Tota } \\
(n=1\end{array}$ & \\
\hline 4 o más & $3(5,7)$ & $1(1,7)$ & $4(3,6)$ & & Media & Rango & Media & Rango & Media & Rango \\
\hline Exacerbaclones graves & & & & Visitas a urgencias por otras causas & 0,53 & 0 a 7 & 0,48 & 0 a 5 & 0,50 & 0 a 7 \\
\hline $\begin{array}{l}0 \\
1\end{array}$ & $\begin{array}{r}28(52,8) \\
8(15,1)\end{array}$ & $10(16,7)$ & $\begin{array}{l}59(52,2) \\
18(15,9)\end{array}$ & Visitas a urgencias por asma & 1,26 & 0 a 12 & 1,78 & 0 a 14 & 1,54 & 0 a 14 \\
\hline 2 & $5(9,4)$ & $4(6,7)$ & $9(8,0)$ & Hospitalizaciones por asma, $\sin$ UCI & 0,36 & 0 a 10 & 0,18 & 0 a 3 & 0,27 & 0 a 10 \\
\hline 4 o más & $\begin{array}{l}5(1,4) \\
7(13,2)\end{array}$ & $13(21,7)$ & $20(17,7)$ & Hospitalizaciones por asma, con UCI & 0,34 & 0 a 10 & 0,08 & 0 а 3 & 0,20 & 0 a 10 \\
\hline Datos expresados como frecuencia & porcentaje) o media ( & (desviación est & tándar) & Hospitalizaciones por otras causas, sin UCI & 0,08 & 0 a 2 & 0,23 & 0 a 6 & 0,16 & 0 a 6 \\
\hline * Basado en indice de masa corpora & , justado para adole & escentes. $n=59$ & en el grupo & Hospitalizaciones por otras causas, con UCI & 0,11 & 0 a 3 & 0,07 & 0 a 1 & 0,09 & 0 a 3 \\
\hline & & & & Exacerbaciones & 1,96 & 0 a 32 & 2,05 & 0 a 14 & 2,01 & 0 a 32 \\
\hline
\end{tabular}

Declaraciones: Este estudio fue patrocinado por GSK, código PRJ2544. Todos los autores son empleados y/o accionistas de GSK.

Referencias

1. Ministerio de Salud y Protección Social, Organización Panamericana de la Salud. Morbi-mortalidad de las enfermedades respiratorias crónicas, Colombia 2005-2013 2016.

2. Neffen H, Moraes F, Viana K, Di Boscio V, Levy G, Vieira C, Abreu G, Soares C. Asthma severity in four countries of Latin America. BMC Pulm Med. 2019; 19: 123.

3. Chung KF, Wenzel SE, Brozek JL, Bush A, Castro M, Sterk PJ, et al. International ERS/ATS guidelines on definition, evaluation and treatment of severe asthma. Eur Respir J. 2014;43(2):343-73. 